\title{
Ein Gerät zur Umsatzbestimmung
}

\section{Zugleich ein Beitrag zur Wärmeleitfähigkeitsmessung der Exspirationsluft

\author{
Von Robert G. A. Lotz
}

Aus dem Zoologischen Institut der Johann-Wolfgang-Goethe-Universität Frankfurt a. M. (Z. Naturforschg. 3 b, 356-361 [1948]; eingegangen am 7. Oktober 1948)

Es wird die Brauchbarkeit der Wärmeleitfähigkeitsmessung zur Ermittlung des Grundumsatzes geprüft.

Durch Diffusion des Meßgases zu den Hitzdrähten, eine Anordnung, die in der industriellen Meßtechnik häufig Anwendung findet, wird erreicht, daß die Messung weitgehend von der linearen Strömungsgeschwindigkeit unabhängig wird. Die Empfindlichkeit der Sauerstoffbestimmung wird gesteigert durch Erhöhung der Hitzdrahttemperatur. Eine Anordnung zur Bestimmung des Grundumsatzes am Menschen wird beschrieben, die es gestattet, im „offenen System“ laufende Messungen durchzuführen. Die „laufende Messung“" wird den integrierenden Methoden gegenübergestellt.

$I^{n}$ n der Klinik ist die Bestimmung des Grundumsatzes zum unentbehrlichen Hilfsmittel geworden. Besonders die Behandlung von hormonalen Störungen, ausgehend von Hypophyse und Schilddrüse, machen eine fortlaufende Úberwachung des Grundumsatzes notwendig.

Zur Bestimmung des Gasstoff wechsels am Menschen oder Tier ist eine große Reihe von Anordnungen und Verfahren bisher beschrieben worden. Die direkte Kalorimetrie oder auch die Respirationskalorimetrie zur Bestimmung des Grundumsatzes kommen für die Praxis kaum in Frage. In beiden Fällen wird die Wärmeabgabe gemessen; bei der Respirationskalorimetrie unter gleichzeitiger Bestimmung des respiratorischen Stoff wechsels. Bei der indirekten Kalorimetrie dagegen wird, in einem „offenen“ oder "geschlossenen" System, der Sauerstoff verbrauch und die gebildete Kohlensäure bestimmt. Im „offenen System" wird der Luftverbrauch gemessen und gasanalytisch die Zusammensetzung der Atemluft festgestellt. Dabei kann durch eine Gasuhr oder auch in einen gasdichten Sack (Douglas) ausgeatmet werden. Für praktisch-medizinische $\mathrm{Zwecke}$ ist wohl z. Zt. am meisten die „Atmung im geschlossenen System" im Gebrauch. Hierzu werden registrierende Spirometer benutzt, die mit Sauerstoff gefüllt werden, wie solche von Krogh, Benedict oder Knipping angegeben wurden. An einer Stelle des Atmungsweges sind dabei Gefäße mit Barytlauge oder Natronkalkfüllung eingeschaltet, die die ausgeatmete Kohlensäure absorbieren. Die Volumabnahme, welche

$1 \mathrm{H}$. Wolls chitt, W. B othe, H. Ruska u. E. G. S c h e n k, Naunyn-Schmiedebergs Arch. exp. Pathol. Pharmakol. 177; 635 [1935].

2 K. F. L u f t, Z. techn. Physik 24, 97 [1943].

3 A. V. H ill, J. Physiology 56, Proc. XX [1922].

4 H. W. K n i p p in g, Hoppe-Seyler's Z. physiol. Chem. 141, 1 [1924].

5 P. G. L e d i g u. R. S. L y m a n n, J. clin. Invest. 4,495 [1927]. das Spirometer registriert, entspricht dem Sauerstoffverbrauch. Die Kohlensäure wird titrimetrisch oder auch, nach Freimachen mit Schwefelsäure aus dem Absorptionsgefä $ß$, volumetrisch bestimmt.

Diese gasanalytischen und spirometrischen Methoden ergeben bei sorgfältiger Durchführung gute Werte. Allerdings benötigen alle einen erheblichen Aufwand an Gerät und setzen eine gewisse Erfahrung bei der Handhabung voraus. Auf bestimmte Ungenauigkeiten, die bei diesen Methoden auftreten, wird später noch eingegangen werden.

Man war daher schon lange bestrebt, diese Verfahren durch einfachere Methoden und die Gasanalyse durch Messung physikalischer Eigenschaften zu ersetzen. Einmal wurde die Änderung des Brechungsvermögens der Atemluft bei wechselnder Zusammẹnsetzung herangezogen ${ }^{1}$. Ein anderes, recht interessantes Verfahren wurde im Laboratorium der I.G. Farbenindustrie, Oppau, entwickelt ${ }^{2}$. Hierbei wird die Ultrarotabsorption zur Gasanalyse benutzt. Die Verminderung der Strahlung durch das zu bestimmende Gas wird durch die selektive Erwärmung eines Gasvolumens mit Hilfe eines. Membrankondensators gemessen. Beide Methoden sind infolge ihres großen apparativen Aufwandes mehr für das wissenschaftliche Laboratorium geeignet und kommen für die ärztliche Praxis kaum in Frage. Die meist sehr hohe Empfindlichkeit dieser Geräte ist von der wirklichen Genauigkeit infolge der Fehlerquellen recht verschieden.

Die meisten physikalischen Methoden jedoch benutzen die Änderung der Wärmeleitfähigkeit bei wechselnder Gaszusammensetzung ${ }^{3-10}$. Hierbei wird

${ }^{6}$ A. K. N o y o n s, Arch. néerl. Physiol. Homme Animaux 7, 488 [1932].

7 E. Tied e mann u. A. Fis che r, Biochem. Z. 247, 68 [1932].

8 H. R e in, Naunyn-Schmiedebergs Arch. exp. Pathol. Pharmakol. 171, 363 [1933].

${ }_{9}$ H. R e in, Hb. biol. Arbeitsmethoden, 1937, Abt. IV, Tl. 13, S. 795 .

10 W. Fill, Zool. Anzeiger 136, 170 [1941]. 
meist die Anordnung von Schleiermacher zugrunde gelegt, da sie die einzige Methode ist, die es gestattet, kontinuierliche Messungen durchzuführen.

Das Prinzip ist folgendes: Die $\mathrm{zu}$ analysierende Luft befindet sich in einem Hohlraum, der von gut wärmeleitendem Material umgeben ist. Durch den Hohlraum geht ein drahtförmiger Leiter, meist Platin, der durch elektrischen Strom erwärmt wird. Die sich an dem Draht einstellende Temperatur ist abhängig von dem Wärmeleitvermögen der Umgebung, also der Luft. Die Drahttemperatur kann demnach als Maß für die Wärmeleitfähigkeit dieser Luft benutzt werden. Die Wärmeleitfähigkeit der Luft ändert sich mit ihrer Zusammensetzung. Da nun der Widerstand des Drahtes von seiner Temperatur abhängig ist, kann dieser als Maß für die Temperatur verwendet werden. So kann also die Messung auf die Bestimmung eines elektrischen Widerstandes zurückgeführt werden. Die genaue Bestimmung des Widerstandes geschieht mit Hilfe der Wheatstoneschen Brückenschaltung. Eine derartig genaue Widerstandsmessung in Wheatstonescher Brückenschaltung setzt voraus, daß ein genau gleich angeordneter Vergleichswiderstand benutzt wird. Praktisch führt man dies so durch, daß man zwei gleich konstruierte und abgeglichene Meßkammern so anordnet, daß die eine mit der zu messenden Luftkomponente, die andere mit einer möglichst ähnlichen Vergleichsluft konstanter Zusammensetzung gefüllt ist. In unserem Fall wird bei der Kohlensäurebestimmung Ausatemluft gegen Frischluft (der die Kohlensäure entzogen wurde), bei der Sauerstoffbestimmung Ausatemluft (ohne Kohlensäure) gegen normale Frischluft gemessen.

R e i $n^{s}$ macht seine Messungen im bewegten Luftstrom, der senkrecht an den Hitzdrähten vorbeigeführt wird. Nach seinen Angaben sind bei dieser Meßanordnung Massendichte und spezifische Wärme des Gases die wesentlichsten Punkte, während die eigentliche Wärmeleitfähigkeit nur eine untergeordnete Rolle spielt. Durch eine solche Anordnung der Hitzdrähte wird die Messung naturgemäß sehr abhängig von der linearen Strömungsgeschwindigkeit der Luft. Die Erhaltung einer konstanten Luftgeschwindigkeit in der Meßzelle erfordert andererseits einen verhältnismäßig großen apparativen Aufwand. In der Technik, in der diese Methode bei den Rauchgasprüfern schon sehr lange Anwendung findet, hat man daher die Hitzdrähte meist so angeordnet, daß die Luft durch Diffusion an den Draht herangebracht wird. Hierdurch wird die Messung durch die lineare Gasgeschwindigkeit kaum beeinflußt11. Es wird also hierbei im wesentlichen nur die veränderte Wärmeleitfähigkeit der Luft gemessen. Voraussetzung bei allen diesen Messungen ist natürlich, daß die Luft vorher vollständig getrocknet wird. Auf Grund der nahezu gleichen Werte des Temperaturkoeffizienten der Wärmeleitfähigkeit von Luft und Sauerstoff bzw. Stickstoff glaubt $\mathrm{R}$ e i $\mathrm{n}^{8}$ keine genügende Empfindlichkeit dér

11 P. G m e lin u. H. G r ü s s, Eucken-Jakob, Der Chemie-Ingenieur, 1933, Bd. II, Tl. IV, S. 86 .
Sauerstoffbestimmung zu erreichen. Diese gleichen Werte des Temperaturkoeffizienten der Wärmeleitfähigkeit, wie sie in physikalisch-chemischen Tabellen meist angegeben werden, gelten bei $0^{\circ} \mathrm{C}$. Versuche von $\mathrm{Tiedemann}$ und $\mathrm{Fischer}{ }^{7}$ sowie von Fill $\mathbf{1 0}^{\mathbf{1 0}}$ haben aber ergeben, daß ein erheblicher Unterschied dieser Werte bei erhöhten Temperaturen besteht.

In den letzten vier Jahren wurden am Zoologischen Institut der Universität Frankfurt a. M. unter Leitung von Prof. Gi ersberg Untersuchungen über den Zusammenhang von Strahlung und Stoffwechsel durchgeführt (s. S. 349). Im Rahmen dieser Untersuchungen ist, aufbauend auf die Methoden zur technischen Gasanalyse und die Apparatur von Fill ${ }^{10}$, ein Gerät weiterentwickelt worden, das sich nicht nur zur Bestimmung des Gasstoffwechsels bei Tieren, sondern auch, nach entsprechenden Veränderungen, zur Umsatzbestimmung am Menschen eignet. Hierbei wurde ebenfalls die Methode der Wärmeleitfähig keitsmessung gewählt, da die Bestimmung anderer physikalischer Konstanten meist nicht so relativ einfach ist. Die Anordnung vereinigt große Empfindlichkeit und Anzeigeschnelligkeit. Die Messungen beanspruchen wenig Zeit, setzen nur geringe Fertigkeit und Geschicklichkeit des Beobachters voraus und verlangen weniger Rechenarbeit oder andere Überlegungen bei der Auswertung, was für die Praxis ausschlaggebend sein dürfte.

Die Anordnung, wie sie für Tiere Anwendung findet, wird im Rahmen einer anderen Arbeit beschrieben werden. Hier möchte ich mich auf die Beschreibung des Apparates, wie er bei der Bestimmung des Grundumsatzes am Menschen benutzt wird, beschränken.

In Abb. 1 ist eine schematische Utbersicht der Anordnung gegeben.* Die Hitzdrähte ${ }_{s}$ sind so angeordnet, daß die Luft durch Diffusion an diese herangebracht wird. Dies wird dadurch erreicht, daß die Luft an Schlitzen vorbeistreift und durch diese zu den Hitzdrähten diffundiert. In Abb. 2 ist eine solche Meßzelle im Querschnitt abgebildet. Eine Senkrechtstellung der Meßkammern ist nicht unbedingt notwendig, da fehlererzeugende Konvektionsströmungen sich dadurch praktisch nicht auswirken können. Die Hitzdrähte aus Platin

* Hrn. Dr. To eller von der Firma Hartmann und Braun A.G., Frankfurt a. M., möchte ich auch an dieser Stelle für seinen Rat und seine Unterstützung danken. 


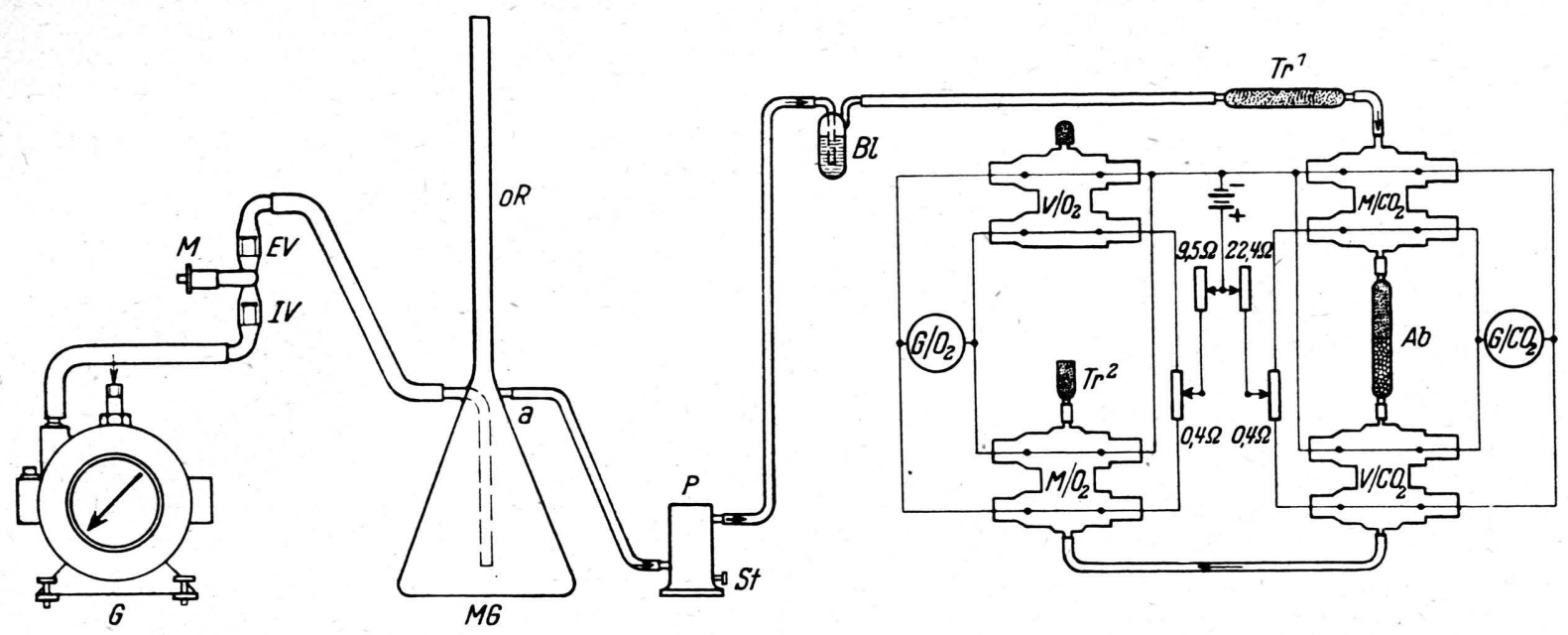

Abb. 1. Schema des Grundumsatz-Meßgerätes. Mafstab 1:10, mit Ausnahme der Meßkammern und der elektrischen Schaltskizze, die vergrößert wiedergegeben sind.

sind $31 \mathrm{~mm}$ lang und haben einen Durchmesser von $0,027 \mathrm{~mm}$. Vier solcher Hitzdrähte sind so angeordnet, daß șich in einer Meßzelle zwei diagonal einander gegenüberliegende Brückenarme befinden. Durch diese bereits von Koepsel ${ }^{12}$ beschriebene Anordnung werden Fehler weitgehend

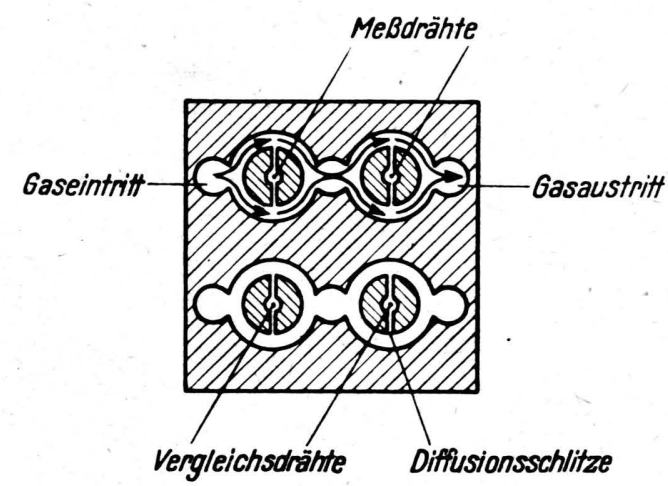

Abb. 2. Querschnitt durch die Meßkammern.

kompensiert und wird die Meßgenauigkeit wesentlich gesteigert. Meßzelle und Vergleichszelle sind in einem gemeinsamen Metallblock untergebracht, wodurch eine gute Wärmeangleichung gewährleistet ist. Um die Empfindlichkeit der Sauerstoffbestimmung zu steigern, wurde die Temperatur der Hitzdrähte wesentlich erhöht (bei besonders genauen Messungen im Tierversuch bis auf $900^{\circ} \mathrm{C}$ ).

Zur Messung des Grundumsatzes atmet die Versuchsperson durch das Mundstück (M). Über das Inspirationsventil (IV) besteht Verbindung zu einem Atemvolumschreiber (G), während von dem Exspirationsventil (EV) eine Leitung zu einem Mischgefäß (MG) von 2-3 Liter Inhalt führt. Hier findet eine Durchmischung der Atemluft statt. Durch ein offenes Rohr (oR) von $60 \mathrm{~cm}$ Länge steht das Mischgefäß mit der Außenluft in Verbindung, wodurch die Atmung vollständig frei im „offenen System“ stattfinden kann. Andererseits ist durch das lange Rohr eine genügende Sicherheit gegeben, daß keine Frischluft von der Pumpe eingesaugt wird. Aus dem Mischgefäß wird (bei a) durch eine elektrische Membranpumpe ständig eine Luftprobe abgesaugt. Diese wird dann durch einen mit konzentrierter Schwefelsäure gefüllten Blasenzähler (Bl) sowie durch eine mit Calciumchlorid gefüllte Trockenröhre $\left(\operatorname{Tr}^{1}\right)$ zu der Meßzelle für die Kohlensäurebestimmung $\left(\mathrm{M} / \mathrm{CO}_{2}\right)$ gedrückt. Nach Austritt aus dieser Meßzelle durchströmt die Luft eine mit Natronasbest und Calciumchlorid gefüllte Absorptionsröhre $(\mathrm{Ab})$ und gelangt in die Vergleichsmeßzelle für die Kohlensäurebestimmung $\left(\mathrm{V} / \mathrm{CO}_{2}\right)$. Schließlich strömt die Luft in die Meßkammer für Sauerstoffbestimmung $\left(\mathrm{M} / \mathrm{O}_{2}\right)$ ein. Den Abschluß bildet eine kleine Trockenröhre $\left(\mathrm{Tr}^{2}\right)$. In der Vergleichsmeßzelle $\left(\mathrm{V} / \mathrm{O}_{2}\right)$ zur Sauerstoffbestimmung ist reine trockene Luft eingeschlossen.

Die Meßgeräte in den beiden Brückenschaltungen sind Galvanometer mit einem Meßbereich von $3 \mathrm{mV}\left(\mathrm{G} / \mathrm{CO}_{2}\right.$ und $\left.\mathrm{G} / \mathrm{O}_{2}\right)$. Außerdem besteht die Möglichkeit, einen Fallbügelschreiber anzuschlieBen, wodurch eine laufende Registrierung mög-

12 A. K o e p s e l, Verh. dtsch. physik. Ges. 10, 814 [1908]; 11, 237 [1908]. 
lich ist. Mit Hilfe von M-illiampèremetern und veränderlichen Widerständen von $9,5 \Omega$ bzw. $22,4 \Omega$ wird der Heizstrom der Hitzdrähte gemessen und reguliert. Als Milliampèremeter dienen die gleichen Galvanometer in den beiden Brücken, welche durch Umschalter entsprechend geschaltet werden können. Die Abgleichung der Brücken geschieht durch Potentiometer von $0,4 \Omega$, mit denen auch der Nullpunkt eingestellt werden kann. Der Heizstrom von insgesamt $525 \mathrm{~mA}$ für die Hitzdrähte wird einem 4-Volt-Konstant-Transformator entnommen. Bei sehr starken Netzschwankungen wird ein 4-Volt-Bleisammler als Puffer zwischengeschaltet.

Die Empfindlichkeit des Gerätes läßt sich durch Veränderung der Nebenschlußwiderstände in der Brückenschaltung variieren. Zur Umsatzbestimmung am Menschen ist sie so eingestellt, daß $3 \mathrm{mV}$ etwa $5 \% \mathrm{CO}_{2}$-Zunahme bzw. $6 \% \mathrm{O}_{2}$-Abnahme entsprechen. Zum Betrieb müssen der Heizstrom für die Meßkammern und die Luftpumpe etwa 30 Min. vor den Messungen eingeschaltet werden, damit eine Wärmeangleichung zwischen Hitzảraht und Kammer stattfinden kann. Andernfalls ändert sich zu Anfang stetig der Nullpunkt. Die Pumpe wird so eingestellt, daß $100-150 \mathrm{~cm}^{3} / \mathrm{min}$ Luft gefördert werden. Schwankungen in der Strömungsgeschwindigkeit bis $\mathrm{zu} \pm 25 \%$ haben keinen Einfluß auf die Messung. Benutzt wird eine elektrische Membranpumpe (P) mit einer Förderleistung bis $500 \mathrm{~cm}^{3} / \mathrm{min}$. Durch eine Stellschraube (St) läßt sich die Förderleistung und damit die Strömungsgeschwindigkeit in weiten Grenzen variieren.

Der Heizstrom wird mit den Widerständen auf $225 \mathrm{~mA}$ für die $\mathrm{CO}_{2}-$ bzw. $300 \mathrm{~mA}$ für die $\mathrm{O}_{2}-\mathrm{Meß}$ kammern eingestellt. Nachdem der Nullpunkt, d. h. also die Frischluftanalyse konstant geworden ist, kann mit der Bestimmung des Gasstoffwechsels begonnen werden.

Zur Bestimmung des Ruhestoffwechsels atmet die Versuchsperson durch eine 10-Liter-Gasuhr (G) ein. Gemessen wird hierbei der Luftverbrauch in der Zeiteinheit. Die Galvanometer für Kohlensäure und Sauerstoff werden ständig abgelesen, bis sie konstante Werte zeigen. Hierbei sind jedesmal der Nullpunkt und die Heizstromstärke neu zu kontrollieren. Die Zeit bis zur Erreichung dieser konstanten Werte kann je nach der Psyche der Versuchsperson recht verschieden sein. Gerade hierin liegt ein großer Vorteil dieser Geräteanordnung, daß man ständig kontrollieren kann, wann normale Atmung stattfindet, die nicht mehr durch nervöse Reizung usw. beeinflußt wird. Bei laufender Registrierung durch einen Fallbügelschreiber läßt sich dieser Zeitpunkt noch leichter festlegen..Die gasanalytischen und spirometrischen Methoden ergeben alle meist dadurch falsche Werte, daß sie über eine längere Zeit integrieren, d. h. also die zu Anfang der Messung durch Unruhe der Versuchsperson meist $\mathrm{zu}$ hohen Werte mit einrechnen. Auch durch eine festgelegte bestimmte „Voratemzeit" läßt sich dieser Fehler nicht ganz ausschalten, wie Versuche ergeben haben. Es gibt Versuchspersonen, die selbst nach über 30 Min. noch keine normale Atmung zeigen. Andere wieder atmen zu Anfang gleichmäßig, um nach 10 oder 15 Min. in ein unnatürliches hastiges Atmen überzugehen.

Die Latenzzeit des Apparates beträgt für $\mathrm{CO}_{2}$ 60 Sek. und für $\mathrm{O}_{2}$ etwa 120 Sek., was für diese Untersuchungen vollauf genügt. Kurzfristige Schwankungen der Raumtemperatur sind zu vermeiden, da sich hierdurch der Nullpunkt ändert. Naturgemäß findet dies ja kaum statt, da ja auch in bezug auf die Versuchsperson eine bestimmte Raumtemperatur eingehalten werden sollte, um Fehler im Grundumsatz auszuschalten.

Die Eichung des Gerätes geschieht entweder durch Ausatemluft, die vorher mit einem HaldaneApparat analysiert wurde, oder, einfacher noch. mit Hilfe eines bekannten Eichgemisches aus Stahlflaschen, das Monate unverändert haltbar ist. Praktischerweise wähit man hierzu ein Gemisch, das einer mittleren Ausatemluft, also einer $\mathrm{Zu}$ sammensetzung von etwa $4 \% \mathrm{CO}_{2}, 16 \% \mathrm{O}_{2}$ und $80 \% \mathrm{~N}_{2}$, entspricht. Die Trocken- und Absorptionsröhren sowie die Schwefelsäure des Blasenzählers sind regelmäßig zu erneuern. Bei ständigem Betrieb wird dies alle 2-3 Tage notwendig sein.

Die Errechnung des Grundumsatzes geschieht in der bekannten Weise. Eine Korrektur der geatmeten Luftmenge, wie dies bei der Bestimmung des Ausatemvolumens notwendig ist, fällt jedoch hierbei weg. Eine Reduktion auf Normaldruck und $0^{\circ} \mathrm{C}$ muß naturgemäß vorgenommen werden. Vom reduzierten Luftvolumen pro Min. wird mit Hilfe der an den Galvanometern abgelesenen $\mathrm{CO}_{2}$ - und $\mathrm{O}_{2}$-Prozente die gebildete $\mathrm{CO}_{2}$-Menge bzw. die verbrauchte $\mathrm{C}_{2}$-Menge in $\mathrm{cm}^{3} / \mathrm{min}$ errechnet. Nach Ermittlung des respiratorischen Quotienten aus diesen Werten wird das kalo- 
rische Aquivalent festgestellt. Hieraus wird dann durch Multiplikation mit dem $\mathrm{O}_{2}$-Verbrauch pro Min. der Grundumsatz errechnet.

Soll der Arbeitsumsatz ermittelt werden, so läßt man in einen Douglas-Sack atmen und schließt diesen dann zur Bestimmung von Kohlensäure und Sauerstoff an die Absaugstelle a (Abb. 1) an. Das Ausatemvolumen, das nach Entleeren des Gummisackes durch eine Gasuhr bestimmt wird, muß in diesem Fall natürlich korrigiert werden.*

* Eine gleiche Apparatur ist, unabhängig von mir, im Kerckhoff-Institut in Bad Nauheim erprobt und auf ihre technischen und biologischen Eigenschaften überprüft worden. H. Gö p f e r t wird seine Erfahrungen über das gesamte Gebiet an anderer Stelle vorlegen.

\title{
Die Temperaturabhängigkeit der Dielektrizitätskonstante von Blut bei Niederfrequenz
}

\author{
Von Hermann Schwan \\ Aus dem Kaiser-Wilhelm-Institut für Biophysik, Frankfurt a. M. \\ (Z. Naturforschg. 3 b. 361-367 [1948]; eingegangen am 23. August 1948)
}

\begin{abstract}
Die im Wellenlängenbereich von 10 bis $1000 \mathrm{~m}$ bestehende Dispersion der DK und Leitfähigkeit biologischen Materials kann auf polare Effekte oder auf durch inhomegene Struktur bedingte Aufladungserscheinungen zurückgeführt werden. Messungen der Temperaturabhängigkeit der DK bei Niederfrequenz ermöglichen die Feststellung der vorliegenden Ursache. In dieser Arbeit wird über die Temperaturabhängigkeit der DK von Blut berichtet. Es ergibt sich, daß, im Gegensatz zur Leitfähigkeit, die DK nicht temperaturabhängig ist und infolgedessen die im mittelfrequenten Gebiet beobachteten Dispersionserscheinungen auf der inhomogenen Struktur des Materials beruhen. Infolge des außerordentlich hohen Verlustfaktors von Blut bei Niederfre uenz ist die Bestimmung der DK von Blut nicht einfach. Ein Teil der Arbeit ist den hierdurch bedingten meßtechnischen Fragen gewidmet.
\end{abstract}

$\mathrm{D}$ ie von einer Reihe von Autoren (Cole, $\mathrm{Cur}$ tis, Fricke, Rajewsky ${ }^{1}$, Osswald u.a.) festgestellte Dispersion der Dielektrizitätskonstante (DK) und Leitfähigkeit biologischen Materials kann auf zwei verschiedenen Ursachen beruhen. Entweder wird sie verursacht durch die Existenz polarer Eigenschaften, oder aber sie beruht auf den durch die inhomogene Struktur der biologischen Substanzen bedingten Nachwirkungserscheinungen. Dänze ${ }^{1}$ hat gezeigt, daß Untersuchungen der Frequenzabhängigkeit der elektrischen Konstanten keinen Aufschluß geben können über die Natur der Dispersionserscheinungen. Es ist daher erforderlich, andere Methoden als die der Frequenzvariation zur Deutung der beobachteten Erscheinungen heranzuziehen. In einer, auf Veranlassung von Rajewsky durchgeführten theoretischen und experimentellen Untersuchung konnte Schwan² zeigen, daß eine solche Möglichkeit in

1 B. Rajewsky, Ergebnisse der biophysikalischen Forschung. Bd. I, Ultrakurzwellen (zusammenfassende Darstellung). Verlag Georg Thieme, Leipzig 1938.

= H. S.chw a n, Ann. Physik V, 40, 509-528 [1941]. der Ermittlung der Temperaturabhängigkeit der DK besteht. Wird nämlich die DK im Gebiet niederer Frequenzen als Funktion der Temperatur bestimmt, so muß sie beim Vorliegen polarer Effekte mit steigender Temperatur abnehmen. Und zwar ist der negative Temperaturkoeffizient um so größer, je größer der Absolutwert der DK ist. Dieses Ergebnis der theoretischen Untersuchungen von $\mathrm{Schw} \mathrm{an}^{3}$ über den Temperaturkoeffizienten der DK von Substanzen mit hohem Temperaturkoeffizienten der DK steht in Übereinstimmung mit den Ergebnissen der im Anschluß daran durchgeführten experimentellen Untersuchungen von Sittel ${ }^{4}$. Da nun aber die DK biologischen Materials, wie weiter unten gezeigt wird, für Niederfrequenz sehr groß ist, müßte der Temperaturkoeffizient der DK beim Vorliegen eines polaren Wirkungsmechanismus

3 H. Schwan, Theorie über das Verhalten von Materialien mit großem Temperaturkoeffizienten der DK in Abhängigkeit von Frequenz und Temperatur. Bisher unveröff.

${ }^{4} \mathrm{~K}$. Sittel, Elektrische Eigenschaften dielektrischer Substanzen mit hohem Temperaturkoeffizienten. Bisher unveröff. 